\title{
Unitarity constraints and role of geometrical effects
}

$\underline{\text { S. M. Troshin, N. E. Tyurin }}{ }^{\mathrm{a}}$

a Institute for High Energy Physics, Protvino, Moscow Region, 142280, Russia

Unitarity and geometrical effects are discussed for photon-photon scattering.

\section{Introduction}

In [1] we have studied limitations off-shell unitarity provides for the $\gamma^{*} p$-total crosssections and geometrical effects in the energy dependence of $\sigma_{\gamma^{*} p}^{t o t}$. It was shown that unitarity by itself does not lead to the saturation at $x \rightarrow 0$, i.e. slow down of the power-like energy dependence of $\sigma_{\gamma^{*} p}^{t}$ and transition to the energy behavior consistent with the Froissart-Martin bound valid for the on-shell scattering. In particular, the $Q^{2}-$ dependence of the constituent quark interaction radius rising with the virtuality $Q^{2}$ leads to an asymptotical result:

$$
\sigma_{\gamma^{*} p}^{t o t} \sim\left(W^{2}\right)^{\lambda\left(Q^{2}\right)}
$$

where $\lambda\left(Q^{2}\right)$ will not depend on virtuality at large values of $Q^{2}$.

Here we consider similar problems for the $\gamma^{*} \gamma^{*}$-scattering. The process of the virtual two-photon scattering is under active study nowadays since it was expected that the hard interaction QCD dynamics would be tested in the most unambiguous way and unitarity would play a minor role there [2]. On the other side there are model approaches which impose saturation in the $\gamma^{*} \gamma^{*}$-interactions, i.e. limitations inherited from a hadronhadron on-shell scattering are extended to this case (cf. [3] and references therein). Such approaches also consider the role of unitarity for the wide range values of virtualities. Available high-energy experimental data obtained at LEP are not restrictive since the data are extracted using Monte-Carlo generators [4].

\section{Unitarity and total cross-sections of real and virtual $\gamma \gamma$-interactions}

Extension of the $U$-matrix unitarization for the off-shell scattering was considered in [6.1]. To apply an extended unitarity to DIS at small $x$ there was supposed that the virtual photon fluctuates into a quark-antiquark pair $q \bar{q}$ and this pair was treated as an effective virtual vector meson state in the processes with small $x$. This effective virtual meson interacts then with a hadron. We considered a single effective vector meson field. To treat $\gamma^{*} \gamma^{*}$-scattering we introduce the amplitude $F_{* *}^{* *}\left(s, t, Q_{1}^{2}, Q_{2}^{2}\right)$ when both initial 
and final mesons are off mass and $F_{*}^{*}\left(s, t, Q_{1}^{2}, Q_{2}^{2}\right)$ when only initial mesons are off mass shell:

$$
V^{*}+V^{*} \rightarrow V^{*}+V^{*}, \quad \text { and } \quad V^{*}+V^{*} \rightarrow V+V
$$

The amplitude $F(s, t)$ describes the on-shell $V V$ scattering.

The unitarity for the amplitudes $F_{* *}^{* *}$ and $F_{*}^{*}$ in impact parameter representation at high energies relates them in the following way

$$
\operatorname{Im} F_{* *}^{* *}\left(s, b, Q_{1}^{2}, Q_{2}^{2}\right)=\left|F_{*}^{*}\left(s, b, Q_{1}^{2}, Q_{2}^{2}\right)\right|^{2}+\eta_{* *}^{* *}\left(s, b, Q_{1}^{2}, Q_{2}^{2}\right),
$$

where $\eta_{* *}^{* *}\left(s, b, Q^{2}\right)$ is the contribution to the unitarity of the many-particle intermediate on-shell states. The solution of the off-shell unitarity relations has a simple form in the impact parameter representation [6]:

$$
\begin{aligned}
F_{* *}^{* *}\left(s, b, Q_{1}^{2}, Q_{2}^{2}\right) & =U_{* *}^{* *}\left(s, b, Q_{1}^{2}, Q_{2}\right)+i U_{*}^{*}\left(s, b, Q_{1}^{2}, Q_{2}^{2}\right) F_{*}^{*}\left(s, b, Q_{1}^{2}, Q_{2}^{2}\right) \\
F_{*}^{*}\left(s, b, Q_{1}^{2}, Q_{2}\right) & =U_{*}^{*}\left(s, b, Q_{1}^{2}, Q_{2}^{2}\right)+i U_{*}^{*}\left(s, b, Q_{1}^{2}, Q_{2}^{2}\right) F(s, b) .
\end{aligned}
$$

The solution of this system has a simple form when the following factorization is imposed

$$
\left[U_{*}^{*}\left(s, b, Q_{1}^{2}, Q_{2}^{2}\right)\right]^{2}-U_{* *}^{* *}\left(s, b, Q_{1}^{2}, Q_{2}^{2}\right) U(s, b)=0 .
$$

Similar factorization was implemented in [1]. It should be noted that these factorization formulas have been implied at the level of an input dynamical quantities and they do not lead to the factorization for the corresponding total cross-sections. Breaking of this factorization is one of the consequences of the unitarity.

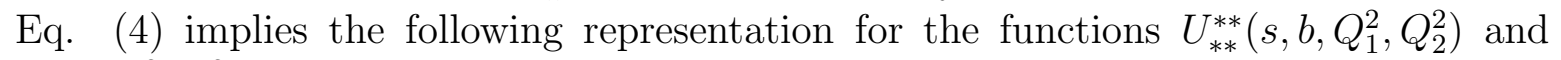
$U_{*}^{*}\left(s, b, Q_{1}^{2}, Q_{2}^{2}\right)$ :

$$
\begin{aligned}
U_{* *}^{* *}\left(s, b, Q_{1}^{2}, Q_{2}^{2}\right) & =\omega^{2}\left(s, b, Q_{1}^{2}, Q_{2}^{2}\right) U(s, b) \\
U_{*}^{*}\left(s, b, Q_{1}^{2}, Q_{2}^{2}\right) & =\omega\left(s, b, Q_{1}^{2}, Q_{2}^{2}\right) U(s, b) .
\end{aligned}
$$

It will be evident in the following that this factorization, in particular, is valid in the off-shell extension of the chiral quark model for the $U$-matrix which we will consider further. The amplitudes $F_{*}^{*}$ and $F_{* *}^{* *}$ then can be written in the form

$$
\begin{aligned}
F_{*}^{*}\left(s, b, Q_{1}^{2}, Q_{2}^{2}\right) & =\frac{U_{*}^{*}\left(s, b, Q_{1}^{2}, Q_{2}^{2}\right)}{1-i U(s, b)}=\omega\left(s, b, Q_{1}^{2}, Q_{2}^{2}\right) \frac{U(s, b)}{1-i U(s, b)} \\
F_{* *}^{* *}\left(s, b, Q_{1}^{2}, Q_{2}^{2}\right) & =\frac{U_{* *}^{* *}\left(s, b, Q_{1}^{2}, Q_{2}^{2}\right)}{1-i U(s, b)}=\omega^{2}\left(s, b, Q_{1}^{2}, Q_{2}^{2}\right) \frac{U(s, b)}{1-i U(s, b)}
\end{aligned}
$$

and unitarity does constraint the magnitudes of the above amplitudes by unity.

The off-shell extension of the model for hadron scattering [5], which uses the notions of chiral quark models was developed in [1]. The further extension for the case when both of the colliding particles (vector mesons) are off mass shell the corresponding $U$-matrix, i.e. $U_{* *}^{* *}\left(s, b, Q_{1}^{2}, Q_{2}^{2}\right)$ should be represented as the product

$$
U_{* *}^{* *}\left(s, b, Q_{1}^{2}, Q_{2}^{2}\right)=\prod_{i=1}^{n_{V_{1}}}\left\langle f_{Q_{i}^{*}}\left(s, b, Q_{1}^{2}\right)\right\rangle \prod_{j=1}^{n_{V_{2}}}\left\langle f_{Q_{j}^{*}}\left(s, b, Q_{2}^{2}\right)\right\rangle .
$$


Factors $\left\langle f_{Q^{*}}\left(s, b, Q_{i}^{2}\right)\right\rangle$ correspond to the individual quark scattering amplitudes smeared over the transverse position of the constituent quark inside the virtual vector meson and over the fraction of longitudinal momentum of the initial parent vector meson. Under the virtual constituent quarks $Q^{*}$ we mean the ones composing the virtual meson.

Further steps are completely similar to the ones described in [1], where the introduction of the $Q^{2}$ dependence into the interaction radius of constituent quark constituent quark (which in the present approach consists of a current quark and the cloud of quarkantiquark pairs of the different flavors [5]) is the main issue of the model.

Dependence on virtuality $Q^{2}$ comes through dependence of the intensity of the virtual constituent quark interaction $g\left(Q^{2}\right)$ and the constituent quark interaction radius $r_{Q^{*}}\left(Q^{2}\right)=\xi\left(Q^{2}\right) / m_{Q^{*}}$ (in the on-shell limit $g\left(Q^{2}\right) \rightarrow g$ and $\xi\left(Q^{2}\right) \rightarrow \xi$ ). The explicit functional dpendencies for the generalized reaction matrices $U_{*}^{*}$ and $U_{* *}^{* *}$ can be written then in the form of (5) with

$$
\omega\left(s, b, Q_{1}^{2}, Q_{2}^{2}\right)=\omega\left(s, b, Q_{1}^{2}\right) \omega\left(s, b, Q_{2}^{2}\right)
$$

and

$$
\omega\left(s, b, Q^{2}\right)=\frac{\left\langle f_{Q^{*}}\left(s, b, Q^{2}\right)\right\rangle}{\left\langle f_{Q}(s, b)\right\rangle},
$$

i. e. in the high-energy limit (for the simplicity we assume here that amplitudes are imaginary and all the constituent quarks have equal masses and parameters $g$ and $\xi$ as well as $g\left(Q^{2}\right)$ and $\xi\left(Q^{2}\right)$ do not depend on quark flavor). Then the functions $U, U_{*}^{*}$ and $U_{* *}^{* *}$ are the following

$$
\begin{aligned}
& U(s, b)=i g^{N}\left(\frac{s}{m_{Q}^{2}}\right)^{N / 2} \exp \left[-\frac{m_{Q} N b}{\xi}\right] \\
& U_{*}^{*}\left(s, b, Q_{1}^{2}, Q_{2}^{2}\right)=\omega\left(b, Q_{1}^{2}\right) \omega\left(b, Q_{2}^{2}\right) U(s, b), \\
& U_{* *}^{* *}\left(s, b, Q_{1}^{2}, Q_{2}^{2}\right)=\omega^{2}\left(b, Q_{1}^{2}\right) \omega^{2}\left(b, Q_{2}^{2}\right) U(s, b),
\end{aligned}
$$

where the function $\omega$ is an energy-independent one and has the following dependence on $b$ and $Q^{2}$

$$
\omega\left(b, Q^{2}\right)=\frac{g\left(Q^{2}\right)}{g} \exp \left[-\frac{m_{Q} b}{\bar{\xi}\left(Q^{2}\right)}\right]
$$

with

$$
\bar{\xi}\left(Q^{2}\right)=\frac{\xi \xi\left(Q^{2}\right)}{\xi-\xi\left(Q^{2}\right)} .
$$

For the on-shell particles $\omega \rightarrow 1$ and we then arrive to the result obtained in [6] at large $s$

$$
\sigma_{\gamma \gamma}^{t o t}(s) \propto \frac{\xi^{2}}{m_{Q}^{2}} \ln ^{2} \frac{s}{m_{Q}^{2}} .
$$


We consider further the off-shell scattering with $\xi\left(Q^{2}\right)>\xi$ and at large $s$ we have

$$
\sigma_{\gamma^{*} \gamma}^{t o t}\left(s, Q^{2}\right) \propto G\left(Q^{2}\right)\left(\frac{s}{m_{Q}^{2}}\right)^{\lambda\left(Q^{2}\right)} \ln \frac{W^{2}}{m_{Q}^{2}},
$$

and for the $\gamma^{*} \gamma^{*}$ total cross-section the following behavior of the total cross-section at large $s$ will take place:

$$
\sigma_{\gamma^{*} \gamma^{*}}^{t o t}\left(s, Q_{1}^{2}, Q_{2}^{2}\right) \propto G\left(Q_{1}^{2}\right) G\left(Q_{2}^{2}\right)\left(\frac{s}{m_{Q}^{2}}\right)^{\lambda\left(Q_{1}^{2}\right)+\lambda\left(Q_{2}^{2}\right)} \ln \frac{s}{m_{Q}^{2}},
$$

where

$$
\lambda\left(Q^{2}\right)=\frac{\xi\left(Q^{2}\right)-\xi}{\xi\left(Q^{2}\right)}
$$

Thus, the steep energy increase of $\gamma^{*} \gamma^{*}$ total cross-section $\sigma_{\gamma^{*} \gamma^{*}}^{\text {tot }} \sim s^{2 \lambda\left(Q^{2}\right)}$ has been predicted at $s \rightarrow \infty$. The $Q^{2}$-dependence of the total cross-sections in the model is determined by the function $G\left(Q^{2}\right)$ which should be chosen on the experimental basis.

The obtained energy dependencies have an asymptotic nature. Existing experimental data obtained at LEP have a significant error bars and do not show clear tendencies as it was mentioned in the Introduction.

Interactions of real and virtual photons are predicted to have significantly different energy dependencies for the total cross-sections.

It is interesting to perform $\gamma \gamma$-studies at higher energies. Tagging two-photon interactions in proton-proton collisions at LHC was considered in [7] and it seems to be experimentally feasible to study $\gamma \gamma$-collisions at high energies.

\section{Acnowledgement}

We are grateful to E. Martynov, V. Petrov and A. Prokudin for the interesting discussions. One of the authors (S. T.) is also grateful to E. De Sanctis and W.-D. Nowak for the support of his participation in European Workshop on the QCD Structure of the Nucleon, Castello Estense, Ferrara, Italy, 3-6 April 2002.

\section{REFERENCES}

1. S. M. Troshin and N. E. Tyurin, Eur. Phys. J. C 22 (2002) 667.

2. S. J. Brodsky, V. S. Fadin, V. T. Kim, L. N. Lipatov and G. B. Pivovarov, CERNTH/2001-341, SLAC-PUB-9069, hep-ph/0111390.

3. N. Timneanu, J. Kwieciński and L. Motyka, TSL/ISV-2001-0253, hep-ph/0110409.

4. A. De Roeck, hep-ph/0101076; G Abbiendi et al. (The OPAL Collaboration), Eur. Phys. J. C 24 (2002) 17.

5. S. M. Troshin and N. E.Tyurin, Nuovo Cim. 106A (1993) 327; Phys. Rev. D 49 (1994) 4427.

6. S. M. Troshin and N. E. Tyurin, Europhys. Lett. 37 (1997) 239.

7. K. Piotrzkowski, Phys. Rev. D 63 (2001) 071502; hep-ex/0201027. 